\title{
Oxygen Uptake in Neonatal Rats: A Developmental Study with Particular Reference to the Effects of Chloramphenicol
}

\author{
Mikko HallmaN ${ }^{[34]}$ \\ Department of Medical Chemistry and Department of Pediatrics, University of Helsinki, Helsinki, Finland
}

\section{Extract}

Chloramphenicol (CHL) treatment in rats begun $0-2 \mathrm{hr}$ after birth inhibited the increase in oxygen uptake, particularly the metabolic response to cold, during the first neonatal days. In rats treated for 5 days with CHL, a few hours after elimination of the drug from the body the tissue levels of ADP and $\mathrm{P}_{\mathrm{i}}$ were significantly higher and the levels of ATP significantly lower than in controls. Furthermore, the presence of more than $0.2 \mathrm{~mm}$ free CHL in serum and liver caused a decrease in oxygen consumption and ATP levels and an increase in $\mathrm{P}_{\mathrm{i}}$ levels. These changes, progressive with increasing CHL concentrations, appeared to be due to direct interference of CHL with mitochondrial electron transport.

Oxygen uptake, as measured in liver slices, increased during the first 2 neonatal days, whereas in slices obtained from adults uptake was lower than in 2-5-day-old animals. In liver slices obtained from rats treated with CHL for 5 days, antimycinsensitive oxygen consumption was deficient both in the presence of excess ADP and in a hypoxic environment.

Oxygen consumption and the steady state reduction levels of cytochrome $c$ were measured in isolated mitochondria with succinate as substrate. According to these studies, the rate-limiting point of oxygen uptake in mitochondria obtained after 5 days of CHL treatment was a deficient phosphorylation site at cytochrome oxidase. On the basis of the previous evidence this was due to selective inhibition of intramitochondrial protein synthesis by CHL.

\section{Speculation}

In rats a rate-limiting point in the neonatal increase in capacity for oxygen uptake is the activity of mitochondrial oxidative phosphorylation. From the resemblances between newborn animals and human infants both in the detrimental effects of CHL and in oxygen uptake in vivo, it seems that the present results can be applied to human development.

\section{Introduction}

During the perinatal period there is an extensive change in the function of the respiratory system. The onset of the function of the lungs is the most dramatic event and poses serious clinical problems. Less is known about the adaptation of cellular respiration to extrauterine life. Cellular respiration occurs mainly in characteristic cytoplasmic membranous structures (mi- 
tochondria). The mitochondrial inner membrane contains the respiratory chain that mediates the reducing equivalents from nutrients via dehydrogenase enzymes to oxygen. This stepwise movement of electrons from one component of the respiratory chain to another releases energy that is used in the process of oxidative phosphorylation to form ATP from ADP and $P_{i}$. Electron transport is coupled to phosphorylation, i.e., the rate of mitochondrial respiration is high in the presence of ADP and $\mathrm{P}_{\mathrm{i}}$ (state 3), whereas excess ATP inhibits oxygen uptake (state 4) [18].

In rat tissues the components of the respiratory chain and the capacity of oxidative phosphorylation increase during the period a few days after birth $[6,11$, $15,17]$. There is evidence that the increase in ambient oxygen tension after birth triggers the mechanism leading to the maturation of the respiratory chain [11].

According to one hypothesis, mitochondria are derived from ancestral bacteria that have adapted to intracellular symbiotic life. Like bacteria, mitochondria contain circular DNA and various species of RNA molecules, and are capable of protein synthesis [3]. Some antibiotics, like CHL, that are effective against bacteria selectively block intramitochondrial protein synthesis in the animal organism. The use of these inhibitors has revealed that intramitochondrial protein synthesis specifically contributes to the formation of the inner membrane and particularly to certain components of the respiratory chain and the machinery of oxidative phosphorylation $[8,13]$.

The aim of this study was to evaluate the role of cellular respiration during perinatal development in the rat. Chloramphenicol was used as a tool to interfere selectively with the development of the inner mitochondrial membrane. The present studies revealed that the disturbed development of the respiratory chain components is of importance in the adaptation of the neonate to extrauterine life.

\section{Experimental Procedure}

Aqueous solution of CHL-sodium succinate [26] was injected into the peritoneal cavity of rats of the Sprague-Dawley strain. This derivative is rapidly hydrolyzed to free CHL in the body. The treatment was started 0-2 $\mathrm{hr}$ after birth as follows: 1st day, 0.62 $\mu \mathrm{mol} / \mathrm{g}$ body wt; $2 \mathrm{nd}$ day, $1.24 \mu \mathrm{mol} / \mathrm{g}$ body wt; $3 \mathrm{rd}-4 \mathrm{th}$ day, $2.48 \mu \mathrm{mol} / \mathrm{g}$ body wt; 5 th day, $3.10 \mu \mathrm{mol} / \mathrm{g}$ body wt. The daily doses were divided into three ( $0-1$ day old), four ( $1-4$ days old), or five (4-5 days old) injections, occurring every 8 th, 6 th, and 4 th- 6 th $\mathrm{hr}$, respectively. The fetal rats used in the slice experiments were obtained by cesarean section. The age of the fetuses was calculated from the gestational period ( $\pm 12 \mathrm{hr}$ ).

ATP, ADP, AMP, pyruvate kinase lactate dehydrogenase, phosphoglycerokinase, myokinase [27], and antimycin A [28] were of analytical grade.

In vivo oxygen consumption was recorded with a laboratory-built, constant pressure respirometer. It was constructed according to the principles described in Handbook of Manometric Techniques [22]. A closed chamber, volume $180 \mathrm{~cm}^{3}$, was connected with a manometer. The atmospheric pressures inside and outside the chamber were equalized with a syringe to within $0.1 \mathrm{ml}$. $\mathrm{NaOH}$ and $\mathrm{CaCl}_{2}$ were used as absorbants of $\mathrm{CO}_{2}$ and water. The maximal capacity for absorption of $\mathrm{CO}_{2}$ and $\mathrm{H}_{2} \mathrm{O}$ was $110 \mu \mathrm{mol} / \mathrm{min}$, but the oxygen uptake during the measurement was always less than $35 \mu \mathrm{mol} \mathrm{O} 2 / \mathrm{min}$. The assay time, i.e., the time when one or two rats were within the chamber, was 3 min for temperature equilibration and $3 \mathrm{~min}$ for the measurement itself. The decrease in oxygen content was linear during the measurement and the loss of oxygen during the assay time never exceeded $15 \%$ of the total oxygen. Between measurements the rats were kept in a humid environment at $35^{\circ}$. During the assays of oxygen uptake in different ambient temperatures, a period between two measurements was 20-30 min (Fig. 3). The measurements proceeded either from lower to higher temperatures or contrariwise. This did not affect the results.

The measurements of temperatures were performed with a thermocouple [29]. The electrode was inserted into the abdominal cavity of rats.

In experiments using liver slices the livers were quickly removed and chilled in an ice-cold incubation medium. Liver slices were prepared with a StadieRiggs tissue slicer [30]. The oxygen consumption of the slices was measured in a Warburg apparatus [22]. The oxygen uptake was linear during the incubation time of $1-2 \mathrm{hr}$.

Mitochondria were prepared by differential centrifugation as described earlier [12].

Oxygen uptake was measured in a closed reaction vessel with magnetic stirring with a Clark-type oxygen electrode [12, 31]. Blood $\mathrm{P}_{\mathrm{O}_{2}}$ was measured with the same oxygen probe. After decapitation of rats the first 1-2 drops of blood were collected in the reaction vessel. The anticoagulant used was $40 \mu \mathrm{l}$ heparin solution in $0.7 \mathrm{ml}$.

The oxidation-reduction levels of cytochrome $c$ and the concentrations of cytochromes $a a_{3}$ and $c$ were meas- 
ured with a dual wavelength spectrophotometer constructed in this laboratory by Dr. I. Hassinen [12].

The determination of CHL was accomplished by the method of Levine and Fisher [19]. Liver and brain were homogenized in $0.25 \mathrm{M}$ sucrose before the assays. In the assays of the half-lives of CHL the single intraperitoneal doses of the antibiotic were $0.31 \mu \mathrm{mol} / \mathrm{g}$ body wt for 0-48-hr-old rats and $0.62 \mu \mathrm{mol} / \mathrm{g}$ body wt for rats older than $48 \mathrm{hr}$. The concentrations of CHL were assayed 1, 2, 4, 6, and $9 \mathrm{hr}$ after the intraperitoneal injection and the half-lives were calculated from a semilogarithmic plot.

$P_{i}$ was measured as described by Ernster et al. [7]. ATP [1], ADP and AMP [2] were determined by the methods of Adam. The liver or heart was immersed in liquid nitrogen within $5 \mathrm{~s}$ after decapitation of the rats, weighed frozen, and homogenized in $0.6 \mathrm{M}$ perchlorate before the assays.

The results are presented as means \pm SE.

\section{Results}

\section{Elimination of Chloramphenicol from Body}

The rate of elimination of CHL from the body is directly proportional to the concentrations of this antibiotic. In the neonate, $\mathrm{t}_{1 / 2}$ for CHL was 6.2 times as high as in the adult. The most rapid increase in the elimination of CHL occurs during the first 2 neonatal days (Fig. 1). Moreover, continuous dosage significantly accelerates the rate of elimination of the antibiotic. This is partly, but not solely, due to the in-

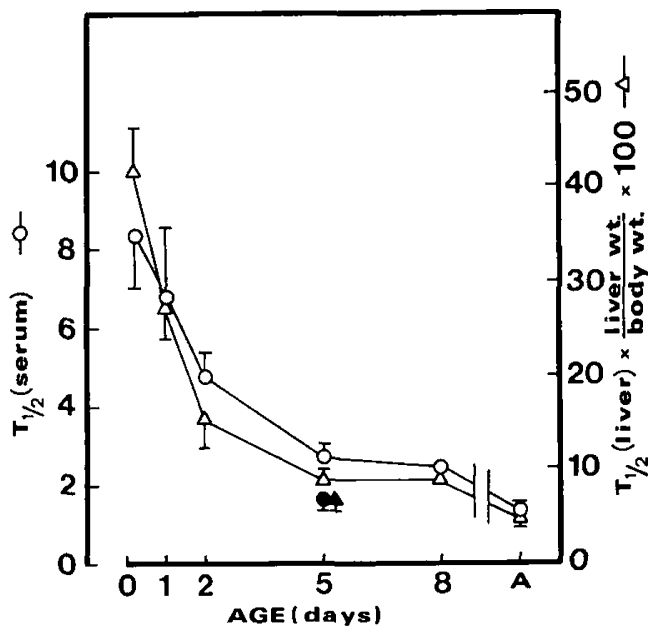

Fig. 1. The half-lives of chloramphenicol ( $\mathrm{t}_{1 / 2}$ in hours) in serum and liver during development. $\bullet, \mathbf{\Delta}$ : experiments with animals chronically treated with chloramphenicol as shown in Experimental Procedure.

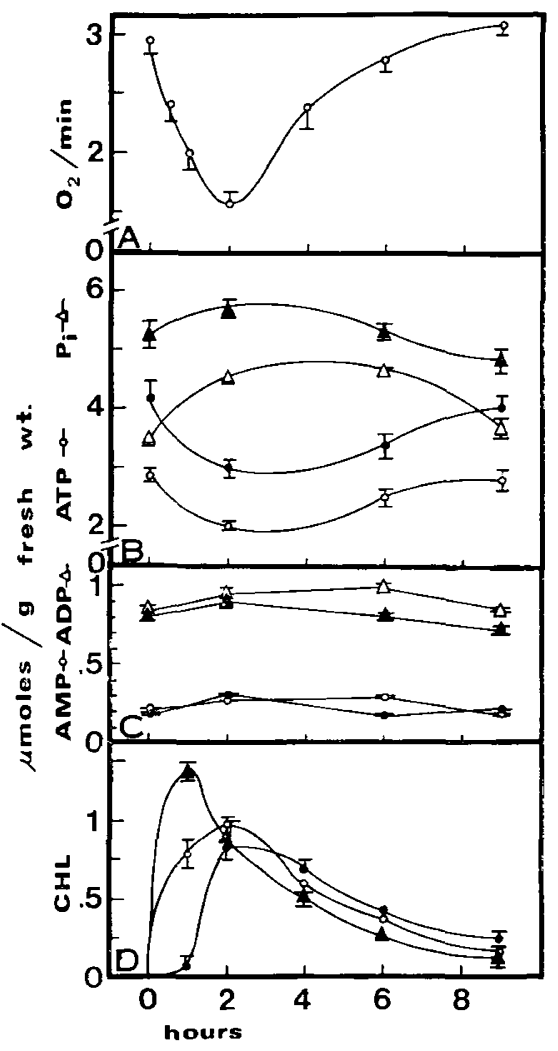

Fig. 2. In vivo oxygen uptake, hepatic and cardiac adenine nucleotide and $P_{f}$ levels, and chloramphenicol (CHL) levels in serum, brain, and liver after single intraperitoneal injection of $1.24 \mu \mathrm{mol}$ chloramphenicol $/ \mathrm{g}$ body wt to 5 -day-old rats. $A$ : measurements of oxygen uptake were performed at an ambient temperature of $21^{\circ}$ (see Experimental Procedure). $B$ and $C$ : assays in liver $(\bigcirc, \triangle)$ and heart $(\bullet, \mathbf{\Delta}) . D: \mathbf{\Delta}$ : serum; $\bullet$ : brain; $\bigcirc$ : liver.

crease of relative liver weight during antibiotic treatment [12].

\section{Effects of Single Chloramphenicol Injection}

The effects of injection of $1.24 \mu \mathrm{mol} / \mathrm{g}$ body $w \mathrm{t}$ intraperitoneal chloramphenicol on the 5-day-old rat are shown in Figure 2. The decreased oxygen consumption paralleled the presence of high concentrations of free CHL in serum and liver. In 0-2-hr-old rats, intraperitoneal injection of $0.41 \mu \mathrm{mol} / \mathrm{g}$ body wt CHL resulted in a prolonged decrease of oxygen consumption, which again paralleled the high levels of CHL in serum and liver (data not shown). Oxygen consumption decreased significantly before measurable amounts of CHL were found in the brain. This suggests that the inhibition of oxygen consumption by CHL is not due to an effect of this antibiotic on the central nervous system.

Adenine nucleotide and $P_{1}$ levels were measured in 
order to evaluate whether CHL inhibits either energy production or energy consumption (Fig. 2). In heart and liver $2 \mathrm{hr}$ after intraperitoneal injection of CHL a significant decrease in ATP and increase in ADP, $A M P$, and $P_{i}$ levels were found. The reversal of these changes largely paralleled the normalization of oxygen consumption. These findings indicate that CHL interferes with the oxidative energy supply of cells.

\section{Effects of Prolonged Chloramphenicol Treatment}

In vivo experiments. The rats treated for 5 days with $\mathrm{CHL}$ had a mortality rate of $52 \% \pm 12 \%$ (control $14 \% \pm 7 \%$ ). The effect of CHL (at the dosage given) on intramitochondrial protein synthesis was tested in each batch of infant rats using liver homogenate and/or isolated mitochondria. The contents of cytochromes $a a_{3}$ and $c$ after CHL treatment for 5 days were $20 \% \pm 2 \%$ and $109 \% \pm 11 \%$ of the contents in untreated rats.

The oxygen uptake of newborn rats was assayed at various environmental temperatures. In Fig. 3 the results for 0-2-hr-old untreated, 5-day-old untreated, and 5-day-old CHL-treated rats are shown. In the last group the measurements were made $7-10 \mathrm{hr}$ after the last injection of CHL, i.e., a few hours after the elimination of the drug from the body. Shortly after birth these animals had a significantly lower oxygen consumption than the older animals. In addition, those treated with CHL showed a significantly lower oxygen consumption at low environmental temperatures than controls of the same age.

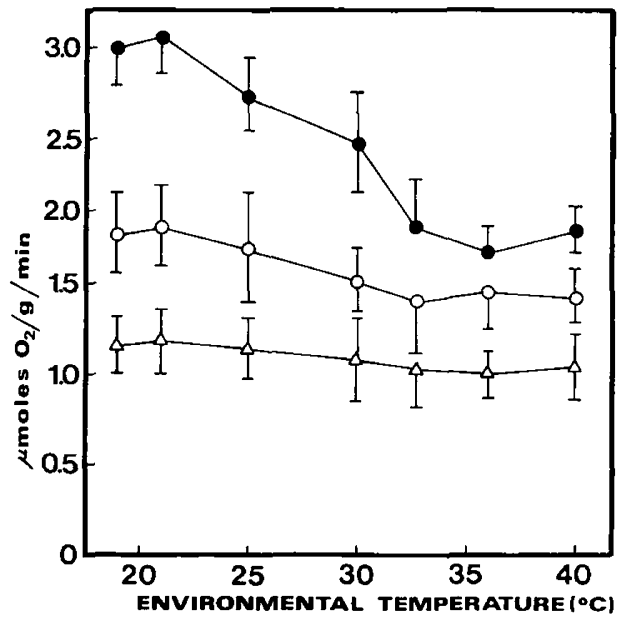

Fig. 3. The oxygen consumption of intact rats. $\triangle: 0-2 \mathrm{hr}$ after birth; . : 5 days after birth; $\bigcirc: 5$ days after birth, chloramphenicol treated.
Several possibilities can be considered as a cause of the differences in oxygen consumption between controls and CHL-treated rats. Anemia caused by CHL may interfere with oxygen transfer. However, hematopoiesis shortly after birth is low, and thereafter the difference in blood hemoglobin between controls (14.4 $\pm 0.5 \mathrm{~g} / 100 \mathrm{ml}$ ) and CHL-treated rats $(13.8 \pm 0.8$ $\mathrm{g} / 100 \mathrm{ml}$ ) was insignificant. Furthermore, the differences in blood $\mathrm{P}_{\mathrm{O}_{2}}$ were not marked: control, $63 \mathrm{~mm}$ $\mathrm{Hg}$; after $\mathrm{CHL}, 69 \mathrm{~mm} \mathrm{Hg}$. The results are means of three experiments. These measurements indicate that the rate of transfer of oxygen to tissues is probably unaffected by CHL.

In human subjects hypothermia has been observed during $\mathrm{CHL}$ treatment. This may reduce oxygen consumption. However, the difference in intraperitoneal temperatures between the two groups of 5-day-old rats was less than $0.7^{\circ}$ in the conditions shown in Fig. 3. The temperatures tended to be lower after CHL treatment (data not shown). Such a small difference in abdominal temperature after CHL treatment is more likely to be the result than the cause of the decreased oxidative metabolism.

The reduced oxygen consumption after CHL treatment might be the result of decreased energy demands. If this were true, no changes would be expected in the adenine nucleotide levels. However, as shown in Table $\mathrm{I}$, the levels of ATP in heart and liver were significantly lower in chronically CHL-treated rats than in untreated rats. In addition, increases were found in the levels of ADP, AMP, and $P_{i}$. This decrease in phosphate potential, ATP/ADP $+\mathrm{P}_{\mathrm{i}}$, suggests a deficiency in the energy-generating system.

Experiments with liver slices. Figure 4 shows the rate of oxygen uptake in liver slices obtained from developing rats. The oxygen consumption increased shortly after birth, but in the adult it decreased again. Addition of ATP inhibited respiration in slices obtained from fetal and 5-day-old rats. However, addition of ATP to slices obtained from adults had no effect on respiration.

In order to differentiate mitochondrial respiration from extramitochondrial respiration, antimycin-sensitive oxygen uptake was measured (Table II). This was always more than $50 \%$ of total respiration. It appeared that in low environmental oxygen tensions antimycinsensitive respiration was significantly lower in slices obtained from CHL-treated rats than in controls. Moreover, in the presence of excess ADP, oxygen uptake was significantly lower after CHL treatment. 
Table I. Effect of chronic chloramphenicol treatment on adenine nucleotide and $\mathrm{P}_{\mathrm{i}}$ levels in heart and liver

\begin{tabular}{|c|c|c|c|c|c|c|}
\hline & ATP & $\mathrm{ADP}$ & & AMP & $P_{i}$ & \multirow{2}{*}{$\frac{\mathrm{ATP}+36 \mathrm{ADP}^{1}}{\mathrm{ATP}+\mathrm{ADP}+\mathrm{AMP}}$} \\
\hline & \multicolumn{5}{|c|}{$\mu \mathrm{mol} / \mathrm{g}$ wet $\mathrm{wt}$} & \\
\hline \multicolumn{7}{|l|}{ Control ${ }^{2}$} \\
\hline Heart & $3.93 \pm 0.20(5)^{3}$ & $0.78 \pm 0.08$ & $(4)$ & $0.19 \pm 0.04$ & $5.32 \pm 0.37$ & 0.88 \\
\hline Liver & $2.71 \pm 0.10$ & $0.89 \pm 0.03$ & (5) & $0.20 \pm 0.02(4)$ & $3.30 \pm 0.31$ & 0.83 \\
\hline \multicolumn{7}{|c|}{ Chloramphenicol } \\
\hline Heart & $3.05 \pm 0.16(10)^{4}$ & $0.83 \pm 0.03$ & $(10)$ & $0.25 \pm 0.02(10)$ & $7.19 \pm 0.37(6)^{\tilde{j}}$ & 0.84 \\
\hline Liver & $2.25 \pm 0.09(6)^{4}$ & $1.11 \pm 0.06$ & $(6)^{6}$ & $0.29 \pm 0.02(6)^{5}$ & $6.01 \pm 0.20(5)^{5}$ & 0.78 \\
\hline
\end{tabular}

1 The energy charge $\left[5_{\lrcorner}^{\circ}\right.$.

${ }^{2}$ Five days old. Decapitation $8 \mathrm{hr}$ after single injection of $0.62 \mu \mathrm{mol} / \mathrm{g}$ chloramphenicol.

${ }^{3}$ Mean $\pm \mathrm{SE}$ (number of experiments appears within parentheses).

${ }^{4} P<0.01$ as compared with the control.

${ }^{5} P<0.05$ as compared with the control.

Experiments with isolated mitochondria. In Figure $5 A$, respiration rates in $C H L$ and control mitochondria are compared. It is shown that in state 3, CHL mitochondria respired more slowly than controls. However, $\mathrm{ADP} / \mathrm{O}$ and the respiratory control ratios were fairly constant. Moreover, uncoupled respiration was only slightly depressed after CHL treatment. Thus, under the present conditions, the deficiencies in the components of the respiratory chain caused by inhibition of intramitochondrial protein synthesis do not seriously limit the electron flow in the respiratory chain.

The content of cytochrome $c$, in contrast to that of the membrane-bound cytochromes of the mitochondrial inner membrane, was unaffected by CHL treatment. Therefore, the steady state levels of reduction of cytochrome $c$ in various respiratory states were measured (Fig. $5 B$ ). Addition of substrate increased the steady state reduction level of cytochrome $c$. Further addition of ADP to CHL mitochondria increased the steady state reduction, which decreased again after the disappearance of ADP. In control mitochondria, on the other hand, the steady state reduction of cytochrome $c$ decreased after addition of ADP. This decrease indicates the release of respiratory control distal to cytochrome $c$. On the other hand, in CHL mitochondria the increase in the steady state reduction level of cytochrome $c$ during state 4-3 transition suggests that the energy transfer distal to cytochrome $c$ is the rate-limiting step in respiration.

\section{Discussion}

In the rat, CHL is either conjugated with glucuronic acid in hepatic microsomes or reduced by nitroreductase in hepatic microsomes and by intestinal tract bac-

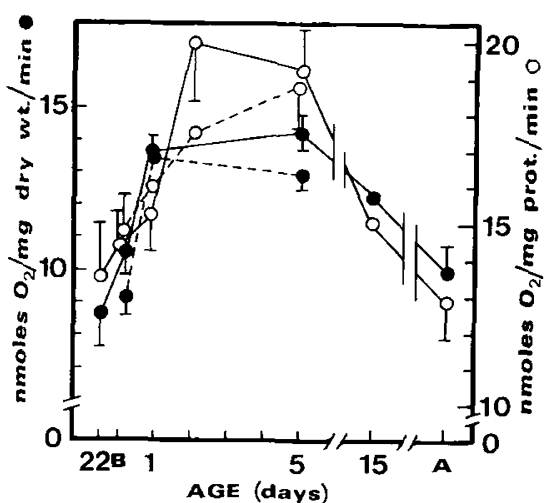

Fig. f. Oxygen uptake in liver slices during development. The slices (150-230 $\mathrm{mg}$ wet wt) were incubated in $2 \mathrm{ml}$ KrebsRinger phosphate (-) or Krebs-Ringer phosphate plus $10 \mathrm{~mm}$ pyruvate and $5 \mathrm{~mm}$ malate $(--)$ at $37^{\circ}$ for $60-120 \mathrm{~min}$ in $100 \%$ oxygen atmosphere. The oxygen consumption was linear after a 15-min equilibration period.

teria [10]. The metabolites produced are excreted in bile and urine. The rate of elimination of free $\mathrm{CHL}$ from serum and liver was low immediately after birth, but increased rapidly during the first 2 neonatal days. Fairly constant levels of CHL were obtained during the postnatal development by careful adjustment of the dosage of this antibiotic. A higher dosage resulted in excessive mortality. On the other hand, with a dosage schedule that allowed the presence of less than 60 $\mu \mathrm{M}$ CHL in the serum between injections, inhibition of intramitochondrial protein synthesis was incomplete [12].

As a result of the inhibition of intramitochondrial protein synthesis, CHL selectively induces a defect in the inner mitochondrial membrane. Certain components of the respiratory chain are drastically decreased in contents, namely cytochromes $a a_{3}$ (cytochrome oxi- 
Table II. Effect of ambient oxygen, ATP, and ADP on antimycin-sensitive oxygen uptake in liver slices ${ }^{1}$

\begin{tabular}{|c|c|c|c|c|c|}
\hline & \multicolumn{5}{|c|}{ Environmental oxygen } \\
\hline & \multirow[t]{2}{*}{$5 \%$} & \multirow[t]{2}{*}{$20 \%$} & \multicolumn{3}{|c|}{$100 \%$} \\
\hline & & & No addition & $\begin{array}{c}\text { Addition of ATP } \\
(10 \mathrm{~mm})\end{array}$ & $\begin{array}{c}\text { Addition of ADP } \\
(10 \mathrm{~m} x)\end{array}$ \\
\hline Fetus, 22 days & & & $9.0 \pm 1.0(7)^{2}$ & $5.7 \pm 0.4(8)^{3}$ & $8.9 \pm 1.0(8)^{3}$ \\
\hline Neonate, 5 days & & & & & \\
\hline Control & $3.1 \pm 0.3(5)^{4}$ & $10.2 \pm 1.3(4)$ & $16.7 \pm 0.6(6)$ & $12.5 \pm 0.6(5)^{5}$ & $16.9 \pm 0.9(6)^{5.6}$ \\
\hline Chloramphenicol & $2.2 \pm 0.2(6)^{4}$ & $9.0 \pm 0.8$ & $16.3 \pm 1.1(7)$ & $14.4 \pm 0.9(7)$ & $14.2 \pm 0.7(7)^{6}$ \\
\hline Adult & & & $8.7 \pm 0.6(6)$ & $8.2 \pm 0.8(6)$ & $8.5 \pm 0.6(6)$ \\
\hline
\end{tabular}

1 Oxygen uptake without antimycin A minus oxygen uptake in the presence of $3 \mu \mathrm{M}$ antimycin A. The incubation medium was KrebsRinger phosphate plus $10 \mathrm{~mm}$ pyruvate and $5 \mathrm{~mm}$ malate.

2 Mean \pm SE (number of experiments appears within parentheses).

${ }^{3} P<0.02$. Footnotes 3 to 6 indicate the degree of significance between the two groups.

${ }^{4} P<0.05$.

${ }^{5} P<0.02$.

${ }^{6} P<0.05$.

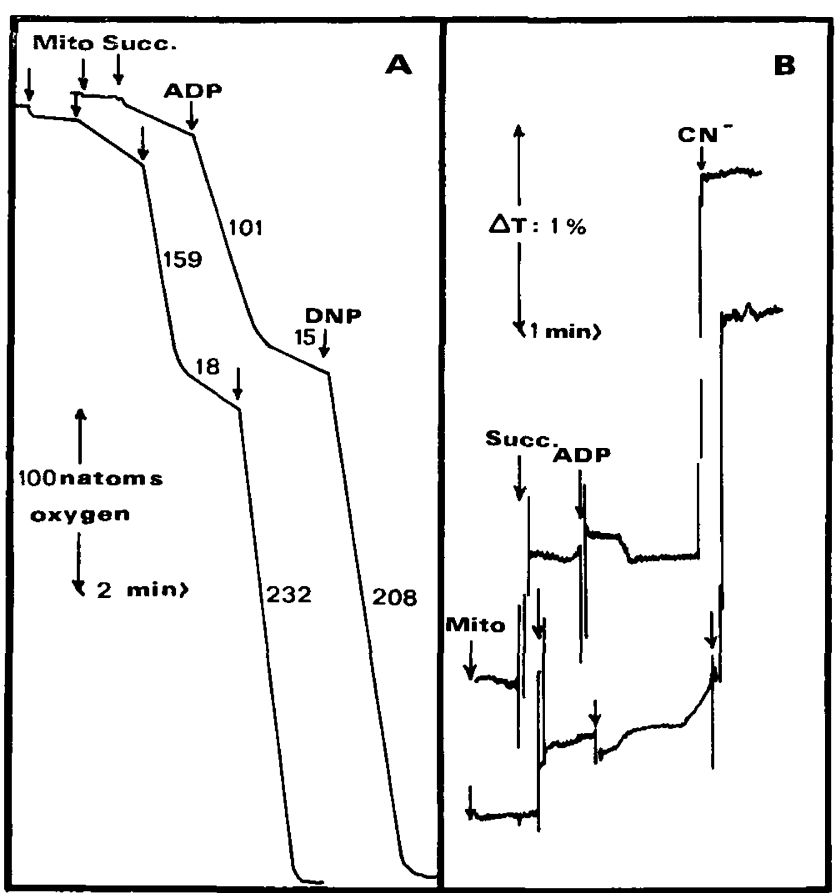

Fig. 5. The effect of chloramphenicol (CHL) treatment on oxygen uptake and steady state reduction levels of cytochrome $c$ in liver mitochondria obtained from 5-day-old rats. The upper graphs represent experiments with mitochondria obtained after chronic CHL treatment. The assay medium was $100 \mathrm{~mm}$ sucrose, $25 \mathrm{~mm}$ Tricine, $7 \mathrm{~mm}$ potassium phosphate, $5 \mathrm{~mm} \mathrm{MgCl}_{\mathfrak{g}}, 30 \mathrm{~mm} \mathrm{KCl}$, $0.5 \mathrm{~mm}$ EDTA, and $2 \mu \mathrm{m}$ rotenone (pH 7.4). $A$ : numbers above the curves indicate respiratory activity in $\mathrm{n}$ atoms oxygen $\times \mathrm{min}^{-1}$ $\mathrm{mg}^{-1}$ protein. The additions were $0.78 \mathrm{mg}$ (CHL) and $0.85 \mathrm{mg}$ (control) mitochondrial protein (Mito), $9 \mu \mathrm{mol}$ succinate (Succ.), $0.2 \mu \mathrm{mol}$ ADP, and $0.15 \mu \mathrm{mol}$ 2,4-dinitrophenol (DNP). Tem. perature $30^{\circ}$; volume $0.82 \mathrm{ml}$. $B$ : difference in transmission at 550 $\mathrm{nm}$ and $535 \mathrm{~nm}$. Additions of $1.58 \mathrm{mg}$ and $1.71 \mathrm{mg}$ mitochondrial protein were used in CHL and control experiments, respectively. The additions were $9 \mu \mathrm{mol}$ succinate (Succ.), $0.2 \mu \mathrm{mol}$ ADP, and $0.7 \mu \mathrm{mol}$ cyanate $\left(C N^{-}\right)$. Temperature $20^{\circ}$; volume $0.7 \mathrm{ml}$. dase) and $b$. Other inner membrane components/activities either decrease (oligomycin-sensitive ATPase) or remain unaffected (cytochrome $c$, succinate dehydrogenase) in contents/activities [8, 12, 13]. These differences reflect the dual origin of the mitochondrial inner membrane components, which are partly synthesized within mitochrondria and partly derived from extramitochondrial sources. There is no convincing evidence that the synthesis of other cellular components is affected by low concentrations of CHL [8, 16, 24]. This experimental approach can be used to study aspects of the formation of the inner mitochondrial membrane and to evaluate the role of the respiratory chain and the activity of oxidative phosphorylation in regulating the energy metabolism of the cell.

In young animals CHL treatment, beginning from birth, decreased the oxygen uptake and the phosphate potentials as measured in liver and heart. This indicates a deficiency in either the transfer of oxygen or cellular oxidative metabolism. No sign of a defective transfer of $\mathrm{O}_{2}$ to tissues was observed, but the finding of deficient oxidative metabolism both in liver slices and in isolated mitochondria is in accord with the latter alternative.

The defective oxygen uptake in liver slices in the presence of low environmental oxygen tension agrees with the finding of decreased activity in mitochondrial cytochrome oxidase [12]. On the other hand, the lack of stimulation of oxygen uptake on addition of ADP after CHL treatment is in accord with the finding of low phosphate potentials in vivo.

Adenine nucleotides affect the activities of several of the enzymes concerned with energy metabolism, namely citrate synthetase, isocitrate dehydrogenase, 
and phosphofructokinase, AMP being the positive and ATP the negative modifier of the respective activities [4]. If these enzymes are rate limiting in the activity of oxygen uptake in the present conditions, CHL treatment appears to inhibit their formation. However, this is unlikely $[8,16]$. On the other hand, the experiments with isolated mitochondria showed that after in vivo CHL the activity of oxidative phosphorylation in the presence of succinate was lower than in the control of the same age. Moreover, the measurements of the steady state oxidation-reduction levels of cytochrome $c$ in isolated mitochondria suggest that the distal portion of the respiratory chain, namely cytochrome oxidase, and, particularly, the phosphorylation site associated with cytochrome oxidase, are factors limiting the rate of oxygen uptake. This finding is in good agreement with experiments in which an uncoupler specific for the energy conservation site in the cytochrome oxidase region was used [13].

The increase in the oxygen uptake of liver slices during the first neonatal days resembled the increase in the contents of the respiratory chain components and the capacity for oxidative phosphorylation. In adults, however, oxygen uptake was disproportionally low as compared with the contents of cytochromes (see Reference 11 ). In addition, there was a variation in the effect of ATP on oxygen uptake: in slices obtained from young animals ATP was inhibitory, but in adults it had no effect. This finding is analogous to the developmental change observed by van Rossum [23] in the effect of an uncoupler of oxidative phosphorylation, dinitrophenol, on oxygen consumption in liver slices. It is tempting to speculate that these findings reflect a developmental change in the factors that control oxygen uptake at the cellular level: in fetal and newborn rats the oxidative phosphorylation associated with the respiratory chain is the rate-limiting point, whereas in adults the capacity for respiration is controlled at a level different from the respiratory chain.

In various mammals, including man, oxygen uptake is lower shortly after birth than a few days later. The present results are in accord with these findings. According to Hill's and Rahimtulla's findings [14], the low metabolic rate of the neonate is independent of the rate of oxygen transfer to tissues. The present results revealed that inhibition in the postnatal development of the respiratory chain enzymes by CHL largely abolished the development of the metabolic response to cold. Therefore, it is plausible to assume that the development of the inner membrane components dur- ing the neonatal period is one important step in the maturation of the mechanism of thermoregulation.

The treatment of newborn and premature infants with CHL in closes exceeding $100 \mathrm{mg} / \mathrm{kg} / 24 \mathrm{hr}$ leads to a toxic reaction called the gray syndrome. The high levels of serum CHL in the gray syndrome (0.2-0.4 $\mathrm{mm}$ ) are due to the deficient detoxification mechanism, probably the low glucuronyl transferase activity, in the hepatic microsomes of the newborn infant $[10,25]$. In the newborn rat the elimination of CHL and tolerance of this drug are deficient, too. It is known that CHL in high concentrations interferes with electron transport in the mitochondrial respiratory chain at site $l$ (i.e., the NADH dehydrogenase portion of the respiratory chain) [9]. The inhibition of oxygen uptake in vivo and the parallel decrease in phosphate potential in the presence of high tissue levels of CHL are in good agreement with these results. Moreover, after $\mathrm{CHL}$ treatment for 5 days beginning a few hours after birth, the capacities for oxygen uptake and oxidative phosphorylation were deficient even after elimination of the antibiotic from the body. This was due to the inhibition of intramitochondrial protein synthesis during the phase of rapid development of the mitochondrial respiratory chain. Thus, it seems that there is deficient production of oxidative energy in the gray syndrome. This is mainly due to direct interference by CHL with the function of the mitochondrial respiratory chain. Secondly, inhibition of the formation of the structures involved in mitochondrial oxidative phosphorylation by $\mathrm{CHL}$, especially in premature newborn infants, may depress oxidative metabolism still further $[c f$. References 12,20$]$.

\section{Summary}

The oxygen consumption of newborn rats was evaluated in intact animals, in liver slices, and in isolated mitochondria. With all three assay methods, the oxygen uptake was shown to increase shortly after birth. Elimination of CHL from the body was low in the neonate, but increased rapidly during the first 2 neonatal days.

There were two mechanisms by which CHL inhibited oxygen uptake. Oxygen consumption decreased when more than $0.2 \mathrm{~mm}$ CHL was present in serum. This was due to direct interference with mitochondrial electron transport. Secondly, the intraperitoneal treatment with CHL for 5 days, beginning shortly after birth, resulted in deficient oxygen uptake. This appears to be due to the defective function of oxidative phosphorylation in mitochondria as a result of inhibi- 
tion of intramitochondrial protein synthesis during the phase of rapid development of the respiratory chain. The present results clarify the role of mitochondrial oxidative phosphorylation in the adaptation of respiration to the extrauterine environment.

\section{References and Notes}

1. Adam, H.: Adenosine-5'-triphosphate: Determination with phosphoglycerate kinase. In: H.V. Bergmeyer: Methods of Enzymatic Analysis, p. 539 (Academic Press, New York, 1963).

2. Adam, H.: Adenosine-5'-diphosphate and adenosine-5'-monophosphate. In: H.-V. Bergmeyer: Methods of Enzymatic Analysis, p. 573 (Academic Press, New York, 1963).

3. Ashwell, M., ANd Work, T. S.: The biogenesis of mitochondria. Annu. Rev. Biochem., 39: 251 (1970).

4. AtKinson, D. E.: Biological feedback control at the molecular level. Science, 150: 851 (1965).

5. Atrinson, D. E.: The energy charge of the adenylate pool as a regulatory parameter: Interaction with feedback modifiers. Biochemistry, 7: 4030 (1968).

6. Dawkins, M. J. R.: Respiratory enzymes in the liver of the newborn rat. Proc. R. Soc. Ser. B. Biol. Sci., 150: 284 (1959).

7. ERnster, L., ZetTerström, R., AND LindberG, O.: A method for the determination of tracer phosphate in biological material. Acta Chem. Scand., 4: 942 (1950).

8. Firkin, F. C., AND LinNane, A. W.: Biogenesis of mitochondria. 8. The effect of chloramphenicol on regenerating rat liver. Exp. Cell Res., 55: 68 (1969).

9. Freeman, K. B., and Haldar, D.: The inhibition of mammalian mitochondrial NADH oxidation by chloramphenicol and its isomers and analogues. Can. J. Biochem., 46: 1003 (1968).

10. Glazko, A. J.: Metabolic aspects of the toxicity of drugs. In: G. L. Hobby: Antimicrobial Agents and Chemotherapy, p. 655 (American Society for Microbiology, 1966).

11. Hallman, M.: Changes in mitochondrial respiratory chain proteins during perinatal development: Evidence of the importance of environmental oxygen tension. Biochim. Biophys. Acta, 253: 360 (1971).

12. Hallman, M.: Effect of intraperitoneal chloramphenicol on some mitochondrial enzymes in neonatal rats. Biochem. Pharmacol., 20: 1797 (1971).

13. Hallman, M., and Hassinen, I.: Effects of uncoupler on rat liver mitochondria isolated after chloramphenicol treatment. Fed. Eur. Biochem. Soc. Lett., 13: 258 (1971).

14. Hill, J. R., and Rahimtulla, K. A.: Heat balance and the metabolic rate of newborn babies in relation to environmen- tal temperature, and the effect of age and of weight on basal metabolic rate. J. Physiol., 180: 239 (1965).

15. Jakovcic, S., Haddock, J., Getz, G. S., Rabinowitz, M., and SwIFT, H.: Mitochondrial development in liver of fetal and newborn rats. Biochem. J., 121: 341 (1971).

16. Kleitke, B., AND Wollenberger, A.: On the site of synthesis of enzymes tightly bound to mitochondrial structure in rat liver. Fed. Eur. Biochem. Soc. Lett., 1: 187 (1968).

17. LANG, C. A.: Respiratory enzymes in the heart and liver of the prenatal and postnatal rat. Biochem. J., 95: 395 (1965).

18. Lehninger, A.: The Mitochondrion, Molecular Basis of Structure and Function (W. A. Benjamin, New York, 1964).

19. Levine, J., AND Fishbach, H.: The chemical determination of chloramphenicol in biological materials. Antibiot. Chemother. 1: 59 (1951)

20. Lietman, P. S.: Pharmacolgic effects on developing enzyme systems. Fed. Proc., 31: 62 (1972).

21. Umbreit, W. W., Burris, R. H., and Stauffer, J. F.: Manometric Techniques, Ed. 4, p. 1 (Burgess Publishing Company, Minneapolis, 1964).

22. Umbreit, W. W., Burris, R. H., and Stacffer, J. F.: Manometric Techniques, Ed. 4, p. 102 (Burgess Publishing Company, Minneapolis, 1964).

23. van Rossum, G. D. V.: Respiration and glycolysis in liver slices prepared from rats of different fetal and postnatal ages. Biochim. Biophys. Acta, 7f: 15 (1963).

24. von Ehrenstein, C., and Lipmann, F.: Experiments on hemoglobin biosynthesis. Proc. Nat. Acad. Sci. L. S. A., 47: 941 (196I).

25. Weiss, C. F., Glazko, A. J., and Weston, J. K.: Chloramphenicol in the newborn infant: A physiologic explanation of its toxicity when given in excessive dose. New Engl. J. Med., 262: 787 (1960).

26. Orion Oy, Helsinki, Finland.

27. Boehringer and Soehne, G.m.b.H., Mannheim. Germany.

28. Sigma Chemical Co., St. Louis, Mo.

29. Ellab Electrolaboratoriet, Copenhagen, Denmark.

30. Arthur H. Thomas Co., Philadelphia, Penn.

31. Model 5331 oxygen probe, Yellow Springs Instrument Company, Yellow Springs, Ohio.

32. I am grateful to Mrs. Maire Ojala and Mrs. Pirkko Erkkilä for their skillful technical assistance and to Associate Professor I. Hassinen, M.D., for critical reading of the manuscript.

33. This research was supported by grants from the Finnish Medical Society Duodecim and the Paulo Foundation.

34. Requests for reprints should be addressed to: Miккo HallMan, M.D., Department of Pediatrics, Lniversity of Helsinki, Stenbackinkatu 11, 00292 Helsinki 29, Finland.

35. Accepted for publication July 3, 1973. 